\title{
Expresión de la metaloproteinasa-9 en pacientes con formas leves y graves de enfermedad por reflujo gastroesofágico
}

\author{
Expression of metalloproteinase-9 in patients with mild and severe forms of \\ gastroesophageal reflux disease
}

\author{
Álvaro J. Montiel-Jarquín ${ }^{1}$, Luis G. Vázquez de Lara-Cisneros², Aurelio López-Colombo ${ }^{*}$, \\ Hugo A. Solís-Mendoza1', María L. Palmer-Márquez y María S. Romero-Figueroa ${ }^{3}$ \\ 'División de Investigación en Salud, Unidad Médica de Alta Especialidad, Hospital de Especialidades de Puebla, Instituto Mexicano del Seguro \\ Social (IMSS), Academia Nacional de Educación Médica en México, Puebla; ${ }^{2}$ Facultad de Medicina, Benemérita Universidad Autónoma de Puebla, \\ Puebla; ${ }^{3}$ Centro de Investigación en Ciencias de la Salud (CICSA), Facultad de Ciencias de la Salud, Universidad Anáhuac, Campus Norte, \\ Huixquilucan. México
}

\begin{abstract}
Resumen
Antecedentes: La enfermedad por reflujo gastroesofágico (ERGE) se desarrolla cuando el contenido estomacal ocasiona síntomas molestos o complicaciones. Las formas leves son esofagitis no erosiva y erosiva; las graves, esófago de Barrett y adenocarcinoma esofágico. Las metaloproteinasas de la matriz degradan componentes de la matriz extracelular, y tienen un papel importante en la invasión tumoral y la metástasis. Objetivo: Relacionar la expresión de la metaloproteinasa-9 (MMP-9) en biopsias esofágicas de pacientes con formas leves y graves de ERGE. Método: Estudio transversal. Se determinó la expresión de MMP-9 en biopsias esofágicas de pacientes con ERGE grave y leve. Las variables fueron edad, sexo, diagnóstico, tabaquismo, alcoholismo, índice de masa corporal (IMC) y expresión de MMP-9. Se realizó estadística descriptiva, concordancia para el diagnóstico y prueba de ji al cuadrado. Resultados: 50 pacientes, 32 (64\%) hombres y 18 (36\%) mujeres, con edad media de $52.13 \pm 14.75$ años. Doce (24\%) fumadores y 7 (14\%) con alcoholismo. El IMC promedio fue de $26.71 \pm 4.07 \mathrm{~kg} / \mathrm{m}^{2}$ (rango: 15-33); 40 (80\%) eran obesos. La concordancia entre observadores para el diagnóstico histopatológico fue de 1.0, y de 0.84 para esofagitis. Veintisiete (54\%) tuvieron esofagitis, 16 (32\%) esófago de Barrett y 7 (14\%) cáncer de esófago. Hubo expresión de MMP-9 en cuatro pacientes con esofagitis, cinco con esófago de Barrett y cinco con cáncer esofágico. Encontramos diferencia estadísticamente significativa entre la expresión de MMP-9 y el tabaquismo $(p=0.011)$ y el diagnóstico histopatológico $(p=0.052)$. Conclusiones: La expresión de MMP-9 es más frecuente en las formas graves que en las leves de ERGE.
\end{abstract}

PALABRAS CLAVE: Adenocarcinoma esofágico. Enfermedad por reflujo gastroesofágico. Esófago de Barrett.Metaloproteinasa-9.

\begin{abstract}
Background: Gastroesophageal reflux disease develops when the stomach contents causes troublesome symptoms and complications. Mild forms are non-erosive and erosive esophagitis, and severe forms are Barrett's esophagus and Esophageal adenocarcinoma. Matrix metalloproteinases are endopeptidases that can degrade components of the extracellular matrix, they play an important role in tumor invasion as well as in metastasis. Objective: To correlate the expression of metalloproteinase 9 (MMP-9) in esophageal biopsies from patients with mild and severe forms of Gastroesophageal reflux disease. Method: Cross-sectional study. The expression of MMP-9 was determined in biopsies of esophageal tissue of patients with mild and severe GRD. The included variables were age, sex, diagnosis, smoking and alcoholic habits, body mass index (BMI)
\end{abstract}

\footnotetext{
Correspondencia:

*Aurelio López-Colombo

2 norte 2004, Col. Centro

C.P. 72000 , Puebla, México

E mail: aurelio.lopezc@imss.gob.mx
}

Fecha de recepción: 01-09-2018

Fecha de aceptación: 13-10-2018

DOI: $10.24875 / C I R U .18000691$
Cir Cir. 2019;87:436-442

Contents available at PubMed www.cirugiaycirujanos.com 
and expression of MMP-9. Descriptive statistics was performed, Kappa for concordance in diagnosis as well as X2. Results: There were 50 patients, $32(64 \%)$ men and 18 (36\%) women, mean age $52.13 \pm 14.75$ years of age. $12(24 \%)$ with smoking and 7 (14\%) with alcoholism. Average BMI was $26.71 \pm 4.07 \mathrm{~kg} / \mathrm{m}^{2}$ (15 to 33); 40 (80\%) with obesity. The inter observer concordance for histopathological diagnosis was 1.0 and 0.84 for esophagitis degrees. 27 (54\%) patients had esophagitis, 16 (32\%) Barrett's esophagus and 7 (14\%) esophageal cancer. There was expression of MMP-9 in four patients with esophagitis, five with Barrett's esophagus and five with esophageal cancer. Statistical significance was found between the expression of MMP9 and smoking $(p=0.011)$ and histopathological diagnosis $(p=0.052)$. Conclusions: The expression of MMP-9 is most common in severe forms compared to the mild forms of GRD.

KEY WORDS: Barret's esophagus. Esophageal adenocarcinoma. Gastroesophageal reflux disease. Metalloproteinase-9.

\section{Introducción}

La enfermedad por reflujo gastroesofágico (ERGE) es una condición que se desarrolla cuando el reflujo del contenido estomacal ocasiona síntomas molestos o complicaciones. Es un padecimiento frecuente, pues el $35-44 \%$ de la población presenta pirosis al menos una vez por mes $^{1,2}$ y el $4-7 \%$ de los individuos aparentemente sanos la padecen de manera cotidia$\mathrm{na}^{3-7}$. Otros estudios han llegado a reportar que hasta un $20 \%$ de la población adulta tiene manifestaciones clínicas debidas a la ERGE ${ }^{8}$. En Europa occidental la prevalencia está en el $10-20 \%$, en Turquía es del $11.9 \%$, en América del Sur es del $10 \%^{2}$ y en Asia es del $6.2 \% \%^{10-15}$.

En el consenso internacional sobre ERGE realizado en Montreal se agruparon los síntomas esofágicos y extraesofágicos ${ }^{2,16,17}$. Así, los casos de síndrome esofágico se dividen en sintomáticos, que son síndromes de reflujo típico y síndrome de dolor torácico por reflujo, y con lesión esofágica, que son esofagitis por reflujo, estenosis por reflujo, esófago de Barrett y adenocarcinoma esofágico; y los casos extraesofágicos se dividen en asociaciones establecidas y asociaciones propuestas. Las asociaciones establecidas son el síndrome de tos por reflujo, el síndrome de laringitis por reflujo, el síndrome de asma por reflujo y el síndrome de erosión dental por reflujo; las asociaciones propuestas son la faringitis, la sinusitis, la fibrosis pulmonar idiopática y la otitis media recurrente.

También en ese mismo consenso se dividió la sintomatología en cuatro grupos:

- Diagnóstico de ERGE basado en la sintomatología: la ERGE puede ser diagnosticada en centros de atención primaria por los síntomas, sin necesidad de que se realicen pruebas adicionales de diagnóstico.

- Dolor torácico: los síntomas de la ERGE pueden ser experimentados en el tórax o el abdomen superior, y pueden ser manifestados como quemazón y dolor. El dolor torácico puede ser parecido al dolor cardiaco isquémico. En estos casos hay que excluir un origen cardiaco del dolor.

- Trastornos serios del sueño: los pacientes con ERGE frecuentemente despiertan durante la noche o son incapaces de conciliar el sueño debido a sus síntomas, que empeoran al estar acostado y mejoran de forma importante con los inhibidores de la bomba de protones.

- Problemas respiratorios: incluyen tos, asma y laringitis. Estos síntomas se asocian a los síntomas clásicos de regurgitación y pirosis, y no mejoran con el tratamiento 2,18,19.

En el año 2002, Fass ${ }^{20-22}$ propuso la categorización de la ERGE únicamente en tres grupos de pacientes: enfermedad por reflujo no erosiva, esofagitis por reflujo erosiva y esófago de Barrett; esto con el fin de enfocar hacia modalidades terapéuticas específicas que beneficiarían a cada grupo individual de pacientes, poniendo la atención sobre la lesión de la mucosa esofágica y los síntomas esofágicos ${ }^{19-22}$. Así, las formas leves fueron la esofagitis erosiva y no erosiva, y las formas graves fueron el esófago de Barrett y el adenocarcinoma esofágico.

\section{Relevancia de las formas graves}

La importancia clínica del esófago de Barrett se deriva de su naturaleza premaligna, ya que tiene el potencial de progresar a adenocarcinoma esofági$\mathrm{Co}^{20-25}$; así mismo, se ha informado de que hasta un $40 \%$ de los pacientes con esófago de Barrett y adenocarcinoma esofágico no presentan síntomas clásicos de reflujo gastroesofágico, condicionando un riesgo aún mayor porque cuando se diagnostican estas complicaciones ya se encuentran en estadios avanzados ${ }^{20,26}$. Estos pacientes requieren la realización de endoscopias seriadas con toma de biopsia, lo cual es económicamente costoso 22 . A pesar de los 
avances en el tratamiento del cáncer esofágico, esta enfermedad maligna tiene una tasa de sobrevida a 5 años menor del $5 \%$, y la fatalidad está relacionada con el estadio en que se diagnostique el tumor. En el estadio I solo se diagnostican un $4 \%$ de los tumores, en el estadio II un $19 \%$, en el estadio III un $21 \%$ y en el estadio IV cerca del $18 \%$. En todo el mundo la tasa de mortalidad es del $90 \%$ de las tasas de incidencia de la enfermedad, y se relaciona con el incremento de la edad ${ }^{27,28}$.

Las metaloproteinasas de matriz (MMP) son una familia de endopeptidasas dependientes de zinc. Colectivamente son capaces de degradar a todos los componentes de la matriz extracelular. Hay evidencia considerable que indica que las MMP tienen un papel importante en la invasión tumoral y la metástasis degradando los componentes de la matriz extracelular y del tejido conectivo circundante a las células tumorales y a la membrana basal. Se ha informado que en los humanos muchos tumores sólidos tienen incrementadas las enzimas proteolíticas, siendo este uno de los pasos clave durante la invasión tumoral y la metástasis. La degradación de las proteínas de la membrana extracelular y la permeabilidad de los vasos sanguíneos son eventos potenciales de las células cancerígenas, llevados a cabo por las MMP, cuya actividad es regulada por inhibidores naturales conocidos como inhibidores tisulares de las MMP. La MMP-9 es una de las gelatinasas que degradan principalmente la colágena tipo IV, que es un componente de la membrana basal de la célula. El uso de inhibidores sintéticos de las MMP ha demostrado una potencial acción inhibitoria de la invasión del cáncer y de las metástasis ${ }^{29}$. Al momento se conocen más de 20 miembros del gen familiar de las MMP humanas, y son clasificadas en subgrupos de colagenasas, estromelisinas y gelatinasas. Esta clasificación se basa en la estructura y la especificidad por el sustrato ${ }^{30}$. La expresión de algunas MMP, como la gelatinasa A (MMP-2), la estromelisina (MMP-3), la matrilisina (MMP-7), la metaloelastasa (MMP-12) y la colagenasa-3 (MMP-13), sugiere la presencia de células tumorales malignas agresivas, lo que condiciona un pronóstico sombrío ${ }^{31}$. En el cáncer colorrectal, la expresión de MMP-9, junto con la de otras metaloproteinasas como MMP-1, MMP-3, MMP-7 y MMP-11, se encuentra elevada en la superficie de las células tumorales. En el cáncer de recto, la expresión alta de MMP-9 no tiene relación con la invasividad, y la MMP7 se expresa en las células cancerosas de los nódulos linfáticos de pacientes con cáncer de recto ${ }^{32}$.
También la MMP-9 y la MMP-2 pueden estar elevadas en otras enfermedades como el cáncer avanzado de ovario $^{33}$, enfermedades inflamatorias como la enfermedad ateromatosa ${ }^{34}$, y complicaciones de la diabetes mellitus ${ }^{34-36}$.

El objetivo de este trabajo es presentar la asociación de la expresión de MMP-9 en pacientes con ERGE en sus formas leves y graves.

\section{Método}

Entre noviembre de 2013 y junio de 2014 se realizó un estudio transversal en el que se comparó la expresión de la MMP-9 en pacientes con esofagitis en sus formas leves y graves, en un centro médico de tercer nivel en la Ciudad de Puebla, en México.

Se incluyeron pacientes con ERGE diagnosticada por el cuestionario de Carlsson Dent, cuya puntuación hubiera sido $\geq 4$, que cumplieron con los criterios de inclusión y aceptaron participar en el estudio mediante el llenado de un consentimiento informado. Decidimos utilizar este cuestionario debido a que con este nivel de corte de 4 puntos tiene una sensibilidad del $70 \%$ y una especificidad del $46 \%$ para padecer esofagitis, y no requiere estudios complementarios ${ }^{1,15,16}$.

Tomando en cuenta que en la literatura se encontró un solo artículo relacionado con la expresión de MMP-9 en patología de esófago, en el que se menciona que alrededor del $40 \%$ de la ERGE en sus formas graves (esófago de Barrett y adenocarcinoma esofágico) presentan expresión alta de MMP-9, en tanto que de los casos leves menos del 10\% la presentan, para una potencia del $80 \%$ y una probabilidad de cometer un error tipo I del $5 \%$, se estima un tamaño de muestra de 21 pacientes por cada grupo, por lo que decidimos llevar el estudio a 52 pacientes. Al momento de realizar los cortes histopatológicos hubo muestra insuficiente en dos, por lo que se eliminaron del estudio y quedaron 50 pacientes.

Se incluyeron pacientes con ERGE en formas leves y graves, de cualquier sexo, de entre 18 y 60 años, que aceptaron participar voluntariamente en el estudio y llenaron el cuestionario de Carlsson Dent para reflujo gastroesofágico. Se excluyeron pacientes con cáncer de tubo digestivo a otros niveles, con datos clínicos de obstrucción de tubo digestivo o con muestra insuficiente por defecto o errores en la toma de esta, y los pacientes con reflujo gastroesofágico pero con resultado histopatológico normal. Las variables consideradas fueron diagnóstico, edad, sexo, tabaquismo, alcoholismo, índice de masa corporal (IMC) 
y expresión de MMP-9 en tejido obtenido por biopsia endoscópica.

Para efectos de este estudio, en diagnóstico se consideró como forma leve la presencia de reflujo sintomático y esofagitis, y como forma grave el esófago de Barrett y el adenocarcinoma esofágico. Se consideró tabaquismo positivo cuando el paciente consumió al menos un cigarrillo, puro o pipa por día; y alcoholismo cuando el paciente refirió el consumo de cuatro onzas de cualquier bebida alcohólica por día. EI IMC se consideró de acuerdo con los criterios de la Organización Mundial de la Salud y se calculó como el peso en kilos por la talla al cuadrado por metro cuadrado; un resultado $\geq 30$ se consideró como obesidad. Se consideró expresión positiva de MMP-9 en la biopsia cuando se observó la coloración característica al microscopio óptico de acuerdo con el fabricante del reactivo.

\section{Procedimiento}

A los pacientes que aceptaron participar en el estudio se les aplicó el cuestionario de Carlsson Dent para ERGE. A los que resultaron con una puntuación $>4$ puntos se les realizó una exploración física completa y se calculó el IMC. Posteriormente se les realizó endoscopia alta con toma de dos biopsias, una para el estudio histopatológico y otra para el estudio inmunohistoquímico.

Para el estudio histopatológico, la conservación de las muestras fue en formaldehído y se diagnosticaron por dos anatomopatólogos distintos. Como las formas graves se presentan con mucha menor frecuencia que las leves $(0.5-1 \% \text { de todas las ERGE) })^{1,19-22}$, cuando se detectó un caso grave se pareó con un caso leve del mismo sexo y edad.

Para el estudio inmunohistoquímico, la conservación de las muestras fue en formol amortiguado (no más de 96 h). Los cortes de los especímenes fueron a menos de cuatro micras y se fijaron en laminillas silanizadas. La desparafinación fue en xileno y la rehidratación se realizó en etanol gradeado. La actividad de la peroxidasa endógena fue bloqueada por incubación durante 30 minutos a temperatura ambiente en peróxido de hidrógeno al $3 \%$. Después de lavar las secciones tres veces en preparación de PBS (Phosphate Buffered Saline) por 5 minutos, se llevó a cabo un bloqueo inespecífico con solución BSA-PBS (Bovine Serum Albumin-Phosphate Buffered Saline) al 1\% por 10 minutos a temperatura ambiente. Las muestras se incubaron con anticuerpos MMP-9 antihumanos monoclonales óptimamente diluidos (clono: 36 020.111, sistema R\&D) a $37^{\circ} \mathrm{C}$ por 60 minutos en una cámara humidificada. Se lavaron tres veces en PBS, llevando a cabo una señal de conversión con el sistema LSAB2 de laboratorios DAKO y de acuerdo con las especificaciones de estos. Finalmente, se realizó la tinción con hematoxilina y se consideró positivo cuando se detectaron anticuerpos adheridos y dieron la coloración característica. Como control interno para la prueba de inmunohistoquímica se usó un caso negativo tomado de un paciente con ERGE sin esofagitis, por cada grupo de muestras.

Se efectuó un análisis descriptivo de los datos, utilizando la media como medida de tendencia central, la desviación estándar como medida de dispersión y la prueba de ji al cuadrado para la comparación de proporciones entre los individuos que expresaron y no expresaron MMP-9 en cada grupo. Se consideró significativa una $p \leq 0.05$.

Este estudio no requirió financiamiento y fue debidamente autorizado por el Comité Local de Investigación y Ética en Investigación en Salud de la unidad médica hospitalaria correspondiente, con número de registro R-2009-2102-46.

\section{Resultados}

Se incluyeron 52 pacientes, de los que fueron excluidos dos debido a que las biopsias, al ser revisadas al microscopio óptico, no tenían tejido suficiente para su interpretación, por lo que quedaron 50 pacientes. La edad promedio fue de $52.13 \pm 14.75$ años (rango: 24-84). De los pacientes estudiados, 12 (24\%) tuvieron antecedente de tabaquismo y 7 (14\%) de alcoholismo. El IMC promedio fue de $26.71 \pm 4.07 \mathrm{~kg} / \mathrm{m}^{2}$ (rango: 15-33); en 40 (80\%) pacientes se detectó obesidad.

Los resultados del estudio histopatológico fueron los siguientes: 27 (54\%) muestras con diagnóstico de esofagitis, 16 (32\%) con esófago de Barrett y 7 (14\%) con cáncer de esófago.

De los 27 (100\%) casos de esofagitis, 12 (44.4\%) fueron esofagitis leve, 9 (33.3\%) esofagitis moderada y $6(22.2 \%)$ esofagitis grave; $16(100 \%)$ pacientes tuvieron esófago de Barrett y, de los 7 (100\%) casos de cáncer esofágico, 5 (71.4\%) fueron adenocarcinoma esofágico y $2(28.6 \%)$ carcinoma epidermoide.

La concordancia intraobservador para el diagnóstico general, esófago de Barrett y tipo de cáncer fue de 1.0, y para los grados de esofagitis fue de 0.84 (kappa). 
Tabla 1. Asociación entre variables sociodemográficas y diagnóstico histopatológico

\begin{tabular}{lcc}
\hline Ji al cuadrado & \multicolumn{2}{c}{ Diagnóstico histopatológico } \\
\cline { 2 - 3 } & $\mathbf{J i}$ al cuadrado & $\mathbf{p}^{*}$ \\
\hline Sexo & 0.196 & 0.907 \\
Tabaquismo & 4.133 & 0.127 \\
Alcoholismo & 2.048 & 0.359 \\
Obesidad & 2.358 & 0.308 \\
\hline
\end{tabular}

*Se consideró estadísticamente significativo un valor de $p \leq 0.05$.

De los 27 pacientes con esofagitis, 17 (62.96\%) eran hombres y $10(37.03 \%)$ eran mujeres. De los 16 pacientes con esófago de Barrett, 10 (62.5\%) eran hombres y $6(37.5 \%)$ eran mujeres. De los 7 pacientes con cáncer esofágico, $5(71.42 \%)$ eran hombres y $2(28.57 \%)$ eran mujeres $(p=0.907)$. Con respecto al hábito de fumar de los pacientes con esofagitis, 9 (33.33\%) fumaron y 18 (66.66\%) no fumaron; de los de esófago de Barrett, 1 (6.25\%) fumó y 15 (93.75\%) no fumaron; y de los portadores de cáncer esofágico, $2(28.57 \%)$ fumaron y $5(71.42 \%)$ no fumaron $(p=0.127)$. Con respecto a la ingesta de alcohol de los pacientes con esofagitis, 4 (14.81\%) ingirieron y $23(85.18 \%)$ no ingirieron bebidas alcohólicas; de los de esófago de Barrett, 1 (6.25\%) ingirió bebidas alcohólicas y $15(93.75 \%)$ no; y de los de cáncer esofágico, $2(28.57 \%)$ ingirieron bebidas alcohólicas y $5(71.42 \%)$ no ingirieron bebidas alcohólicas $(p=0.359)$. Con respecto a la obesidad, de los pacientes con esofagitis, 7 (25.92\%) fueron obesos y 20 (74.07) no tuvieron obesidad; de los de esófago de Barrett, 3 (18.75\%) fueron obesos y $13(81.25 \%)$ no; y de los de cáncer esofágico, ninguno tuvo obesidad $(p=0.308)$ (Tabla 1).

La expresión de MMP-9 fue positiva en 8 (16\%) hombres y en $6(12 \%)$ mujeres, y negativa en $24(48 \%)$ hombres y $12(24 \%)$ mujeres $(p=0.529)$. Con respecto al fumar, fue positiva en 6 (12\%) fumadores y $8(16 \%)$ no fumadores, y negativa en $6(12 \%)$ fumadores y $30(60 \%)$ no fumadores $(p=0.052)$. Con respecto a los que tuvieron ingesta de alcohol, fue positiva en $4(8 \%)$ de los que tomaban y en $10(20 \%)$ de los que no tomaban bebidas alcohólicas, y negativa en $3(6 \%)$ de los que tomaban y en $33(66 \%)$ de los que no tomaban $(p=0.064)$. Con respecto a la obesidad, fue positiva en $1(2 \%)$ obeso y en $13(26 \%)$ no obesos, y negativa en 9 (18\%) obesos y 27 (54\%) no obesos $(p=0.156)$. En cuanto a los resultados del diagnóstico histopatológico y la expresión de MMP-9,
Tabla 2. Asociación entre variables sociodemográficas y expresión de la metaloproteinasa-9 en pacientes con cáncer de esófago

\begin{tabular}{lcc}
\hline Ji al cuadrado & \multicolumn{2}{c}{ MMP-9 } \\
\cline { 2 - 3 } & Ji al cuadrado & $\mathbf{p}^{*}$ \\
\hline Sexo & 0.397 & 0.529 \\
Tabaquismo & 3.791 & 0.052 \\
Alcoholismo & 3.429 & 0.064 \\
Obesidad & 2.358 & 0.308 \\
Diagnóstico histopatológico & 8.961 & 0.011 \\
\hline
\end{tabular}

*Se consideró estadísticamente significativo un valor de $p \leq 0.05$.

de los pacientes con esofagitis, 4 (14.8\%) tuvieron expresión de MMP-9; de los pacientes con Esófago de Barrett, 5 (31.2\%) tuvieron expresión de MMP-9; y de los pacientes con cáncer esofágico, 5 (71.4\%) tuvieron expresión de MMP-9 $(\mathrm{p}=0.011)$ (Tabla 2).

\section{Discusión}

En la literatura mundial existe muy poca información sobre la relación de la expresión de la MMP-9 y las formas graves de ERGE. Herszenyi, et al. ${ }^{37}$ publicaron un estudio en población húngara, en el que reportan valores altos de expresión de MMP-9 dentro de la membrana citoplasmática y del citoplasma del epitelio esofágico en las células displásicas de pacientes con la secuencia de ERGE-esofagitis-esófago de Barrett-displasia-adenocarcinoma esofágico. Incluyeron siete pacientes con esófago de Barrett y ocho con adenocarcinoma esofágico, y encontraron una expresión mayor de MMP-9 en estos pacientes comparados con la expresión de MMP-9 en el epitelio esofágico normal $(p<0.05)$. Sin embargo, algunos autores difieren con respecto a la existencia de esta secuencia ERGE-esofagitis-esófago de Barrett-displasia-adenocarcinoma esofágico ${ }^{20-23}$. El esófago de Barrett típicamente progresa de metaplasia con atipia a metaplasia con displasia y adenocarcinoma. La identificación correcta y temprana de los cambios con un alto riesgo de transformación maligna, como displasia de alto grado y adenocarcinomas tempranos, en pacientes con esófago de Barrett es de gran importancia clínica, ya que ofrece mayor oportunidad de curación ${ }^{20,25}$. Se han propuesto diferentes marcadores para ayudar a una mejor determinación del riesgo para el desarrollo de cáncer, pero muchos de estos marcadores biológicos necesitan una evaluación más profunda ${ }^{37}$. 
Para tener una mejor certeza diagnóstica, sometimos las 50 muestras a evaluación por dos anatomopatólogos distintos, y obtuvimos una excelente concordancia entre observadores (kappa: 1.0). Con respecto al sexo de los pacientes incluidos en el estudio, encontramos una proporción hombre: mujer de 3:1, lo cual concuerda con lo descrito en la literatu$\mathrm{ra}^{35,38}$. La edad promedio de presentación fue menor que en otras series ${ }^{17,29}$, y el porcentaje de pacientes con hábito tabáquico y alcohólico fue bajo, del 24 y el $14 \%$, respectivamente, lo que también concuerda con lo reportado por otros autores $31,34,35$.

La edad promedio para los pacientes que presentaron adenocarcinoma fue de 49.85 años, mucho menor que la reportada en otras series anglosajonas y caucásicas, que reportan una edad de 65 años ${ }^{29,37}$.

La población con formas graves de ERGE fue mucho menor que la población con formas leves. Esto se debe a que, como se ha demostrado, las formas graves constituyen solo un $0.4-1 \%$ de la ERGE ${ }^{17,19,22}$. La cuenta de formas graves de ERGE en nuestro estudio coincide con la del estudio de Herszenyi, et al. ${ }^{37}$ realizado en población Húngara, a pesar de que en Hungría se reporta una incidencia aún más baja de formas graves de ERGE que la reportada en el mundo.

En este estudio se encontró una asociación entre la expresión de MMP-9 y las formas graves de ERGE, con una expresión de MMP-9 baja en las formas leves. Estos hallazgos concuerdan con la expresión de MMP-9 descrita por Herszenyi, et al. ${ }^{37}$, quienes encontraron mayor expresión en las formas graves de ERGE que en las formas leves dentro de la secuencia de ERGE como esofagitis-esófago de Barrett sin displasia-esófago de Barrett con displasia-adenocarcinoma esofágico. Sin embargo, esta secuencia no se ha demostrado, ya que no hay ningún estudio sobre el seguimiento de los pacientes diagnosticados con formas leves hasta el desarrollo de formas graves ${ }^{19,21}$. Grimm, et al. ${ }^{38}$ encontraron que la MMP-1 desempeña un papel como factor preinvasivo en el adenocarcinoma esofágico asociado a esófago de Barrett. También Samantaray, et al. ${ }^{40}$, Mukherjee, et al. $^{41}$ y Ohashi, et al. ${ }^{41}$ encontraron que los valores altos de MMP-9 se encuentran asociados con la variedad escamosa de cáncer de esófago.

En este estudio también se halló una asociación positiva entre la expresión de MMP-9 y la presencia de tabaquismo ( $p=0.052$ ), así como el diagnóstico histopatológico ( $p=0.011$ ), pero no así para la expresión de MMP-9 y el sexo, el alcoholismo y la obesidad ( $p=0.529, p=0.064$ y $p=0.308$, respectivamente), considerando que se debe tener una mayor precaución en los pacientes con hábito tabáquico positivo y en los que tienen diagnóstico histopatológico de esófago de Barrett y adenocarcinoma esofágico. Así mismo, Samantaray, et al. ${ }^{39}$ encontraron una correlación positiva entre la expresión de MMP-9 y el cáncer escamoso y el sexo. Una de las debilidades de este estudio es que la determinación de la expresión de MMP-9 en biopsias fue cualitativa.

Según estos resultados, se concluye que la expresión positiva de MMP-9 está asociada a las formas graves de ERGE, como son el esófago de Barrett y el adenocarcinoma esofágico. Por ello se sugiere el seguimiento de estos pacientes con nuevas mediciones de MMP-9 para poder asociarla con otros riesgos y con la supervivencia de los pacientes.

\section{Conflicto de intereses}

Los autores declaran no tener conflicto de intereses.

\section{Financiamiento}

Este trabajo fue financiado por el Fondo para la Investigación en Salud del Instituto Mexicano del Seguro Social, con número FIS/IMSS/PROT/MD10/867.

\section{Responsabilidades éticas}

Protección de personas y animales. Los autores declaran que los procedimientos seguidos se conformaron a las normas éticas del comité de experimentación humana responsable y de acuerdo con la Asociación Médica Mundial y la Declaración de Helsinki.

Confidencialidad de los datos. Los autores declaran que han seguido los protocolos de su centro de trabajo sobre la publicación de datos de pacientes.

Derecho a la privacidad y consentimiento informado. Los autores han obtenido el consentimiento informado de los pacientes y/o sujetos referidos en el artículo. Este documento obra en poder del autor de correspondencia.

\section{Bibliografía}

1. Montiel-Jarquín AJ. Expresión de la metaloproteinasa 9 en pacientes con formas leves y graves de enfermedad por reflujo gastroesofágico. [Tesis de Maestría en Ciencias Médicas e Investigación, BUAP-IMSS]. Puebla, México; 2011.

2. Uscanga L, Nogueira-de-Rojas JR, Gallardo E, Bernal-Reyes R, González M, Ballesteros-Amozurrutia A. Gastroesophageal reflux disease. Gastroenterology Mexican association consensus. Mexican group for the GERD study. Rev Gastroenterol Mex. 2002;67:216-23. 
3. Vakil N, van Zanten SV, Kahrilas P, Dent J, Jones R: [The Montreal definition and classification of gastroesophageal reflux disease: a global, evidence-based consensus paper]. Z Gastroenterol. 2007;45:1125-40.

4. El-Serag HB. Time trends of gastroesophageal reflux disease: a systematic review. Clin Gastroenterol Hepatol. 2007;5:17-26.

5. Locke GR, III, Talley NJ, Fett SL, Zinsmeister AR, Melton LJ, III. Prevalence and clinical spectrum of gastroesophageal reflux: a population-based study in Olmsted County, Minnesota. Gastroenterology. 1997:112:1448-56.

6. Nebel OT, Fornes MF, Castell DO. Symptomatic gastroesophageal reflux: incidence and precipitating factors. Am J Dig Dis. 1976;21:953-6.

7. Thompson WG, Heaton KW. Heartburn and globus in apparently healthy people. Can Med Assoc J. 1982;126:46-8.

8. Wienbeck M, Barnert J. Epidemiology of reflux disease and reflux esophagitis. Scand J Gastroenterol Suppl. 1989;156:7-13.

9. López-Colombo A, Morgan D, Bravo-González D, Montiel-Jarquín A, Méndez-Martínez S, Schmulson M. The epidemiology of functional gastrointestinal disorders in Mexico: a population-based study. Gastroenterol Res Pract. 2012;2012:606174.

10. Peralta-Pedrero ML, Lagunes-Espinosa AL, Cruz-Avelar A, Juárez-Cedillo T, Rodríguez-Moctezuma R, López-Carmona JM, et al. [Frequency of gastroesophageal reflux disease in elderly patients attending a family medicine clinic]. Rev Med Inst Mex Seguro Soc. 2007:45:447-52.

11. Wong WM, Lai KC, Lam KF, Hui WM, Hu WH, Lam CL, et al. Prevalence, clinical spectrum and health care utilization of gastro-oesophageal reflux disease in a Chinese population: a population-based study. Aliment Pharmacol Ther. 2003;18:595-604.

12. Hui TT, Fass SM, Giurgiu DI, lida A, Takagi S, Phillips EH. Gastroesophageal disease and nausea: does fundoplication help or hurt? Arch Surg. 2000;135:545-9.

13. Armstrong D, Marshall JK, Chiba N, Enns R, Fallone CA, Fass R, et al. Canadian Consensus Conference on the management of gastroesophageal reflux disease in adults - update 2004. Can J Gastroenterol. 2005;19:15-35. 12.

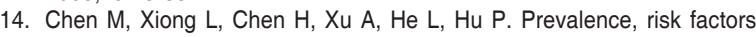
and impact of gastroesophageal reflux disease symptoms: a population-based study in South China. Scand J Gastroenterol. 2005:40:759-67.

15. Klauser AG, Schindlbeck NE, Muller-Lissner SA. Symptoms in gastro-oesophageal reflux disease. Lancet. 1990;335:205-8.

16. Lim SL, Goh WT, Lee JM, Ng TP, Ho KY. Changing prevalence of gastroesophageal reflux with changing time: longitudinal study in an Asian population. J Gastroenterol Hepatol. 2005;20:995-1001.

17. Segal I. The gastro-oesophageal reflux disease complex in sub-Saharan Africa. Eur J Cancer Prev. 2001;10:209-12.

18. Agreus L, Svardsudd K, Talley NJ, Jones MP, Tibblin G. Natural history of gastroesophageal reflux disease and functional abdominal disorders: a population-based study. Am J Gastroenterol. 2001:96:2905-14.

19. Flook N, Jones R, Vakil N. Approach to gastroesophageal reflux disease in primary care: putting the Montreal definition into practice. Can Fam Physician. 2008;54:701-5.

20. Fass R, Fennerty MB, Vakil N. Nonerosive reflux disease - current concepts and dilemmas. Am J Gastroenterol. 2001;96:303-14.

21. Fass R, Hell RW, Garewal HS, Martinez P, Pulliam G, Wendel C, et al. Correlation of oesophageal acid exposure with Barrett's oesophagus length. Gut. 2001;48:310-3.

22. Fass R, Ofman JJ. Gastroesophageal reflux disease - should we adopt a new conceptual framework? Am J Gastroenterol. 2002;97:1901-9.

23. Fass R, Sampliner RE. Barrett's oesophagus: optimal strategies for prevention and treatment. Drugs. 2003;63:555-64.

24. Kerkhof M, van Dekken H, Steyerberg EW, Meijer GA, Mulder AH, de Bruïne A, et al. Grading of dysplasia in Barrett's oesophagus: substantial interobserver variation between general and gastrointestinal pathologists. Histopathology. 2007;50:920-927.

25. Sampliner RE. Updated guidelines for the diagnosis, surveillance, and therapy of Barrett's esophagus. Am J Gastroenterol. 2002;97:1888-95.

26. Pedrazzani C, Catalano F, Festini M, Zerman G, Tomezzoli A, Ruzzenente A, et al. Endoscopic ablation of Barrett's esophagus using high power setting argon plasma coagulation: a prospective study. World J Gastroenterol. 2005;11:1872-5.

27. Munitiz V, Martínez de Haro LF, Ortiz MA, Ruiz de AD, Molina J, Bermejo J, et al. Surgical treatment of high-grade dysplasia in Barrett's esophagus. Cir Esp. 2007:82:214-8.

28. Pickens A, Orringer MB. Geographical distribution and racial disparity in esophageal cancer. Ann Thorac Surg. 2003;76:S1367-9.

29. Van CE, Vantrappen G. Epidemiology and clinical aspects of esophageal cancer. J Belge Radiol. 1991;74:365-8.

30. Yamamoto H, Vinitketkumnuen A, Adachi Y, Taniguchi H, Hirata T, Miyamoto $\mathrm{N}$, et al. Association of matrilysin-2 (MMP-26) expression with tumor progression and activation of MMP-9 in esophageal squamous cell carcinoma. Carcinogenesis. 2004;25:2353-60.

31. Gu ZD, Chen KN, Li M, Gu J, Li JY. Clinical significance of matrix metalloproteinase-9 expression in esophageal squamous cell carcinoma. World J Gastroenterol. 2005;11:871-4.

32. Shima I, Sasaguri Y, Kusukawa J, Yamana H, Fujita H, Kakegawa T, et al. Production of matrix metalloproteinase-2 and metalloproteinase-3 related to malignant behavior of esophageal carcinoma. A clinicopathologic study. Cancer. 1992;70:2747-53.

33. Liabakk NB, Talbot I, Smith RA, Wilkinson K, Balkwill F. Matrix metalloprotease 2 (MMP-2) and matrix metalloprotease 9 (MMP-9) type IV collagenases in colorectal cancer. Cancer Res. 1996;56:190-6.

34. Kamat AA, Fletcher M, Gruman LM, Mueller P, Lopez A, Landen CN, Jr. et al. The clinical relevance of stromal matrix metalloproteinase expression in ovarian cancer. Clin Cancer Res. 2006;12:1707-14.

35. Galis ZS, Sukhova GK, Lark MW, Libby P. Increased expression of matrix metalloproteinases and matrix degrading activity in vulnerable regions of human atherosclerotic plaques. J Clin Invest. 1994;94:2493-503.

36. Beranek M, Kolar P, Tschoplova S, Kankova K, Vasku A. Genetic variations and plasma levels of gelatinase $A$ (matrix metalloproteinase-2) and gelatinase $B$ (matrix metalloproteinase-9) in proliferative diabetic retinopathy. Mol Vis. 2008;14:1114-21.

37. Herszenyi L, Hritz I, Pregun I, Sipos F, Juhasz M, Molnar B, et al. Alterations of glutathione S-transferase and matrix metalloproteinase-9 expressions are early events in esophageal carcinogenesis. World $\mathrm{J}$ Gastroenterol. 2007;13:676-82

38. Grimm M, Lazariotou M, Kircher S, Stuermer L, Reiber Ch, Höfelmayr A, et. al. MMP-1 is a (pre-) invasive factor in Barrett-associated esophageal adenocarcinomas and is associated with positive lymph node status. J Translational Med 2010;8:99.

39. Kollarova H, Machova L, Horakova D, Janoutova G, Janout V. Epidemiology of esophageal cancer - an overview article. Biomed Pap Med Fac Univ Palacky Olomouc Czech Repub. 2007;151:17-20.

40. Samantaray S, Sharma R, Chattopadhyaya TK, Dattta S, Gupta R. Increased expression of MMP-2 and MMP-9 in esophageal squamous cell carcinoma. J Cancer Res Clin Oncol. 2004;130:37-44.

41. Mukherjee S, Roth MJ, Dawsey SM, Yan W, Rodríguez-Canales J, Erickson HS, et al. Increased matrix metalloproteinase activation in esophageal squamous cell carcinoma. J Translational Med 2010;8:91.

42. Ohashi K, Nemoto T, Nakamura K, Nemori R. Increased expression of matrix metalloproteinase 7 and 9 and membrane type 1-matrix metalloproteinase in esophageal squamous cell carcinomas. Cancer. 2000;88:2201-9. 